\title{
Matas de gramíneas como refugios de artrópodos invernantes en agroecosistemas pampeanos: efectos del tamaño, del agrupamiento y de la arquitectura de las plantas
}

\author{
María E. CÁnepa ${ }^{\bowtie, 1}$; Gulllermo A. Montero ${ }^{1} \&$ Ignacio M. Barberis ${ }^{1,2}$ \\ 1. Facultad de Ciencias Agrarias. Universidad Nacional de Rosario. 2. Instituto de Investigaciones en Ciencias Agrarias de \\ Rosario (IICAR). Consejo Nacional de Investigaciones Cientificas y Técnicas, Santa Fe, Argentina.
}

\begin{abstract}
Resumen. Numerosas especies de artrópodos invernantes de la región pampeana utilizan a las gramíneas perennes como refugio. Las características estructurales y el tamaño de las plantas, así como su disposición espacial, condicionarían la disponibilidad de hábitat o de refugio y, en consecuencia, la selección de esas plantas por artrópodos invernantes. En este trabajo evaluamos la influencia del tamaño, el agrupamiento y la modificación de la arquitectura de plantas de Schizachyrium condensatum sobre la abundancia y la riqueza de artrópodos invernantes en su interior. En un borde de un área agrícola de la Pampa Ondulada se dispusieron plantas de distinto tamaño, desde $110 \mathrm{~cm}$ de altura (plantas grandes) a $70 \mathrm{~cm}$ de altura (plantas pequeñas), de manera aislada o en grupos de cinco plantas, a las que se les modificó, o no, la arquitectura (abiertas o cerradas). Luego de la temporada invernal se extrajeron los artrópodos y se determinó la abundancia y la riqueza de morfoespecies en cada planta. Dos especies depredadoras de la familia Coccinellidae, Hippodamia convergens y Coccinella ancoralis, representaron $90 \%$ de la abundancia total, y se concentraron en su mayoría en las plantas grandes agrupadas-cerradas. En las plantas grandes cerradas se registró más del doble de la abundancia de artrópodos que en las plantas grandes abiertas. En las plantas pequeñas no hubo efectos debido a la arquitectura. Se registró similar riqueza de especies de artrópodos entre plantas pequeñas aisladas y plantas pequeñas agrupadas. En las plantas grandes agrupadas se detectó casi el doble de especies de artrópodos que en las plantas grandes aisladas. Estos resultados muestran que el tamaño pequeño, así como la apertura y el aislamiento de las plantas grandes, influyen de forma negativa sobre la selección de los sitios de refugio de coccinélidos invernantes. En consecuencia, planteamos la importancia de la conservación de las gramíneas perennes en los bordes de áreas agrícolas como refugios que asegurarían la permanencia de coccinélidos afidófagos en las proximidades de los campos de cultivo durante el período invernal.
\end{abstract}

[Palabras clave: tamaño de planta, Coccinellidae, hábitat, refugio invernal, tamaño de parche]

\begin{abstract}
AвSTRACt. Tussock grasses as shelters for overwintering arthropods in Pampean agroecosystems: effects of plant size, clustering and architecture: Perennial grasses are used as overwintering shelters by several arthropod species in the Pampa region. Plant size, structure, and clustering may condition habitat or shelter availability, and therefore their selection by overwintering arthropods. We analyzed the influence of clustering, size and architecture modification of Schizachyrium condensatum plants on the abundance and richness of these arthropods dwelling inside these plants. We set up plants of different sizes, from large $(110 \mathrm{~cm}$ tall) to small $(70 \mathrm{~cm}$ tall), along the edge of a field crop in the Rolling Pampas. They were planted isolated or in groups of five plants, with or without architecture modification (open or closed). After the winter season, overwintering arthropods were removed and the abundance and richness of morphospecies were calculated for each plant. Two predator species from the Coccinellidae family, Hippodamia convergens and Coccinella ancoralis, represented almost $90 \%$ of total abundance in large, grouped and closed plants. In large closed plants there was almost twice the abundance of arthropods than in large open plants. In small plants there was no effect due to architecture. In small plants there was similar arthropod richness between isolated and grouped plants, whereas in large plants there was almost twice the species in grouped than in isolated plants. Our results show that small plant size, as well as opening and isolation for large plants, negatively affected the choice of wintering sites for coccinelids. Therefore, we highlight the importance of conserving perennial grasses in the edges of agricultural areas as they allow the persistence of aphidofagous coccinelids in the surroundings of crop fields during wintering periods and thus contribute to the sustainability of the beneficial fauna.
\end{abstract}

[Keywords: plant architecture, Coccinellidae, habitat, winter shelter, patch size]

\section{INTRODUCCIÓN}

En regiones de clima templado, donde sobrevivir el invierno representa un gran desafío para los animales de sangre fría, algunas especies de artrópodos atraviesan una etapa de diapausa reproductiva invernal

Editora asociada: Adriana Ruggiero

mariaeucanepa@yahoo.com.ar
(Flannagan et al. 1998; Musolin \& Numata 2004; Danks 2005; Monetti et al. 2008). Durante este período, los artrópodos utilizan diversos tipos de refugios, como cortezas semi-desprendidas de árboles y arbustos, gramíneas cespitosas, hojarasca u otros residuos vegetales, cuevas de animales y nidos de aves que han sido

Enviado: 14 de julio de 2014, Fin de arbitraje: 9 de octubre, última versión revisada: 20 de marzo de 2015, Aceptado: 29 de marzo. 
abandonados, entre otros (Bucher 1974; Rizzo 1976; Cervantes et al. 2003; Honek et al. 2007; Turienzo \& Di Iorio 2007, 2008).

En la región pampeana, el paisaje invernal es muy homogéneo ya que una proporción elevada de la superficie está cubierta por lotes agrícolas en barbecho o con cultivos como trigo o colza, los que no serían adecuados como refugios invernales para la mayoría de los artrópodos (Montero 2008). Sin embargo, permanecen algunos ambientes con vegetación espontánea que aportan heterogeneidad y conectividad espacial al paisaje, y que son utilizados como sitios de reposo invernal (Altieri 1992; Altieri \& Nicholls 1999; Zaccagnini et al. 2007; Montero 2008, 2014). Por ejemplo, se ha registrado la presencia de diversas especies de artrópodos invernantes debajo de ritidomas de plantas leñosas (Bulacio et al. 2005), en nidos abandonados de aves (Bucher 1974; Turienzo \& Di Iorio 2007, 2008) y en plantas de gramíneas cespitosas presentes en bordes de cultivos y banquinas (Rizzo 1976; Montero 2008). En estos microhábitats, los invernantes más frecuentes son los adultos de coccinélidos (Coleoptera: Coccinellidae) y de chinches fitófagas (Heteroptera: Pentatomidae) (Rizzo 1976; Montero 2008).

En adultos de varias especies de coccinélidos afidófagos se ha registrado "agregación hipsotáctica" (Hagen 1962; Hodek \& Honek 1996). Este mecanismo ocurriría cuando un estímulo quimiotáctico o tigmotáctico activa la producción de ciertos compuestos químicos que actúan como feromonas de agregación durante la comunicación intraespecífica de los individuos (Susset et al. 2013; Wheeler \& Cardé 2013). Debido a esto, los individuos migran en forma gregaria desde las áreas donde se alimentan y se reproducen hacia los sitios de invernación seleccionados (por lo general, matas compactas de gramíneas), donde se protegen de las fluctuaciones fuertes de temperatura y humedad e incrementan la termorregulación grupal (Stephens et al. 1999; Cervantes et al. 2003; Honek et al. 2007). En consecuencia, estas adaptaciones fisiológicas y de comportamiento son de gran importancia ya que favorecen el establecimiento masivo de artrópodos y aumentan la probabilidad de supervivencia de los individuos en el período invernal (Honek et al. 2007).

Las diferencias estructurales entre plantas de distintas especies generan microhábitats con distintas condiciones ambientales que pueden afectar la densidad y la diversidad de artrópodos que las habitan (Lawton 1983; Stuntz et al. 2002; Langellotto \& Denno 2004; Romero \& Vasconcellos-Neto 2005). A su vez, la disposición espacial de las plantas puede conducir a la formación de parches densos de vegetación que son buscados por los artrópodos para encontrar refugios invernales (Hagen 1962; Cervantes et al. 2003). Finalmente, el tamaño de las plantas de una misma especie condicionaría la disponibilidad de hábitat o de refugio para numerosas especies de artrópodos (Cervantes et al. 2003). Asimismo, podrían darse interacciones entre estos factores. Por ejemplo, si bien las matas compactas de mayor tamaño aportarían una mayor disponibilidad de hábitat para los artrópodos que las más pequeñas, una modificación de la arquitectura de la planta podría reducir o anular las diferencias en disponibilidad de hábitat entre plantas de distinto tamaño (Yanes et al. 1982; Honek et al. 2007). No obstante, los efectos del tamaño de la planta, de las modificaciones en la estructura de las plantas, de la disposición espacial de las mismas, así como sus interacciones, aún no han sido evaluados sobre ensambles de artrópodos invernantes en plantas de gramíneas cespitosas de la región pampeana.

En este trabajo evaluamos el efecto de las características del refugio sobre la abundancia y la riqueza de insectos invernantes. Para ello, realizamos un experimento en el que modificamos la arquitectura (abierta o cerrada) y la disposición espacial (aisladas o agrupadas) de plantas de distinto tamaño de "paja colorada» Schizachyrium condensatum (Kunth) Nees. Al igual que otras especies del mismo género, esta gramínea megatérmica C4 florece y fructifica entre enero y marzo, y durante la estación invernal permanece en reposo (Peichoto 2010). En el área de estudio, esta gramínea cespitosa perenne se distribuye principalmente en banquinas de caminos y en bordes de lotes de producción (Peichoto 2003) $\mathrm{y}$ ha sido registrada como sitio de refugio invernal para artrópodos de interés agrícola (Montero 2008). Predecimos que las plantas de mayor tamaño, agrupadas y no modificadas (i.e., plantas cerradas), caracterizadas por presentar mayor disponibilidad de recursos de invernación (e.g., mayor cantidad de hábitats o refugios disponibles y mayor complejidad en la estructura física del microhábitat interno) y por estar más protegidas de variables climáticas adversas y de depredadores, aumentarán la probabilidad de supervivencia de los individuos en el 
período invernal y tendrán mayor abundancia y riqueza de artrópodos invernantes que las plantas pequeñas, aisladas, cuya arquitectura ha sido modificada (i.e., plantas abiertas) y que presentan características opuestas a las anteriores; por lo tanto, se alterarían las condiciones del hábitat donde invernan estos artrópodos.

\section{MATERIALES Y MÉTODOS}

\section{Área de estudio}

El área de estudio posee temperaturas típicas de un clima templado, con veranos cálidos e inviernos suaves. El período libre de heladas, de 275 días, abarca desde principios de septiembre hasta principios de junio. La precipitación media durante el período 1973-2000 fue $987 \mathrm{~mm}$ /año (Sacchi et al. 2002).

\section{Diseño experimental}

A mediados de abril de 2008 se seleccionaron 120 plantas de tamaño mediano a grande de $S$. condensatum en un área deprimida próxima a un cultivo de soja, en las afueras de la ciudad de Rosario (32 $57^{\prime}$ S y 60³9’ W; 23 m s.n.m.), provincia de Santa Fe, Argentina. Al momento de seleccionar las plantas del borde registramos en las mismas la presencia de varias especies de artrópodos de diversos grupos taxonómicos (Armadillidae, Blattodea, Pentatomidae, etc.). Todos los individuos presentes fueron removidos de forma manual antes de trasladar las plantas al área experimental, por lo que las matas no habrían tenido macroartrópodos al iniciarse el experimento. Las plantas fueron trasplantadas individualmente junto con su pan de tierra en macetas plásticas de $5 \mathrm{~L}$ de capacidad. A principios de mayo, estas macetas fueron trasladadas al Campo Experimental J.F. Villarino de la Facultad de Ciencias Agrarias, Universidad Nacional de Rosario, ubicado en la localidad de Zavalla (330.' S y 605' W; 50 m s.n.m.), provincia de Santa Fe, Argentina. A esa altura del año, la mayoría de los lotes de soja de ese área de la Pampa Ondulada ya han sido cosechados y los insectos se encuentran en plena movilidad o iniciando la fase de prediapausa invernal (Monetti et al. 2008).

El área experimental estaba ubicada en el borde de una parcela de producción, rodeada por un área de clausura con vegetación espontánea (Bocanelli et al. 1999) y por lotes con rastrojo de soja y maíz. En una superficie de $5 \times 60 \mathrm{~m}$ se ubicaron de forma sistemática 40 puntos, dispuestos en dos hileras distanciadas a tres metros entre sí (Figura 1). Se seleccionaron al azar 20 puntos en los que se ubicó una planta aislada y en los otros 20 puntos se dispuso una planta rodeada por otras cuatro para formar un grupo o parche (Figura 1). Al momento de la plantación no se removió la vegetación adyacente para ninguno de los tratamientos. La misma era rala y estaba distribuida de manera similar alrededor de todas las plantas trasplantadas. Antes de la asignación de los tratamientos se seleccionaron 40 plantas y se les midió el perímetro y la altura. Las plantas fueron asignadas al azar a los tratamientos de agrupamiento (aislada vs. en grupo), de manera estratificada de acuerdo a su altura. Las plantas en grupo estaban separadas a unos $25-30 \mathrm{~cm}$ entre sí. Consideramos que no habría habido competencia por luz entre las plantas dado que son de crecimiento estival, ni tampoco competencia radical dado que las plantas estaban en macetas individuales. Todas estas macetas fueron enterradas al ras del suelo. El tratamiento de modificación de la arquitectura de la planta fue asignado al azar a la mitad de los sitios de cada tratamiento de agrupamiento (Figura 1). En el centro de estas plantas se colocó un aro de plástico de $5 \mathrm{~cm}$ de diámetro sujeto a los tallos, de forma tal que abriera la planta (Figura 1). Esto se realizó

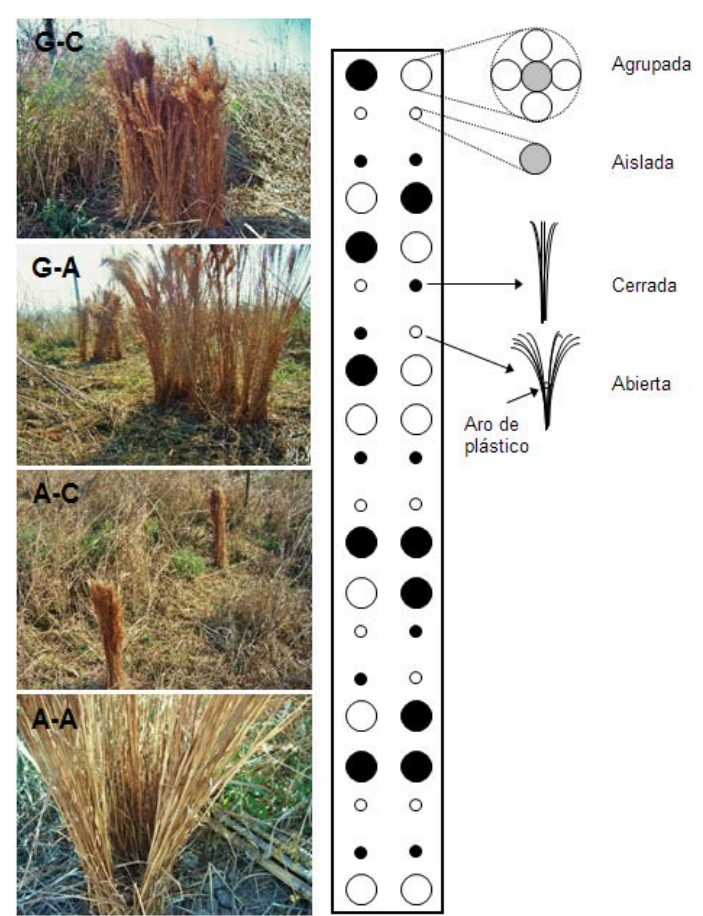

Figura 1. Imágenes y esquema de los distintos tratamientos realizados para variar la arquitectura y la disposición de plantas de S. condensatum en el experimento. G-C: plantas en grupo con arquitectura cerrada, G-A: plantas en grupo con arquitectura abierta, A-C: plantas aisladas con arquitectura cerrada y A-A: plantas aisladas con arquitectura abierta. Los círculos mayores indican grupos de plantas y los círculos menores plantas aisladas. Los círculos blancos muestran plantas de arquitectura abierta y los negros plantas cerradas.

Figure 1. Photographs and scheme of $S$. condensatum plants allocated to the different architectural and clustering treatments in the experiment. G-C: clustered plants with closed architecture, G-A: clustered plants with open architecture, A-C: isolated plants with closed architecture, and A-A: isolated plants with open architecture. Larger circles indicate groups of plants and smaller circles indicate isolated plants. The white circles show plants of open architecture and the black ones closed plants. 
para simular una planta cuya arquitectura ha sido disturbada por algún agente externo (e.g., pisoteo del ganado). Las plantas sin modificación de su arquitectura fueron denominadas plantas cerradas. Todas las plantas permanecieron expuestas a las condiciones ambientales durante 123 días (desde el 02/05/08 al 02/09/08).

\section{Procesamiento de las muestras}

En la primera quincena de septiembre, a las 40 plantas seleccionadas se les cortó la parte aérea al nivel del suelo. El material fue colocado en bolsas de celofán cerradas herméticamente y conservadas en heladera a $5{ }^{\circ} \mathrm{C}$ hasta su procesamiento. Los artrópodos se separaron manualmente y se conservaron en recipientes con alcohol al 70\% para su determinación posterior. El material se agrupó al nivel específico o como morfoespecies diferenciables y se clasificó en familias y órdenes. Cada especie/morfoespecie fue asignada a los siguientes grupos tróficos: detritívoros, herbívoros, depredadores y parasitoides (Root 1973; Hawkins \& Mac Mahon 1989; Farías et al. 2007). Los nombres de los taxones superiores se consignaron según Borror et al. (1992), y para géneros y especies se siguió la clasificación usada por Morrone \& Coscarón (1998). Para cada una de las 40 plantas procesadas se calculó la abundancia y la riqueza de morfoespecies de artrópodos. Luego de extraídos los artrópodos, el material vegetal se secó en estufa a $70^{\circ} \mathrm{C}$ hasta obtener un peso constante y la materia seca se pesó en una balanza de precisión (Scaltec SBA 52, d=0.01 g, Heiligenstradt).

\section{Análisis de datos}

Para evaluar si existían diferencias estadísticas significativas en biomasa, altura y perímetro entre las plantas asignadas a los distintos tratamientos de agrupamiento y arquitectura se realizaron modelos lineales generales (Análisis de Varianza) (Crawley 2007). Para cada una de las variables evaluadas se controlaron los supuestos de normalidad y homogeneidad de la varianza (Crawley 2007). Los análisis estadísticos fueron realizados con la función glm del paquete Rcmdr del programa R (versión 3.1.0; The R Foundation for Statistical Computing 2014).

Para evaluar los efectos del tamaño de la planta, del agrupamiento y de la arquitectura sobre la abundancia y riqueza de morfoespecies de artrópodos se utilizaron modelos de regresión lineal generalizados con distribución de Poisson, incluyendo variables categóricas (agrupamiento y arquitectura) y cuantitativas continuas (biomasa aérea de las plantas) (Crawley 2007).

Si bien la biomasa aérea fue medida al finalizar el experimento, el crecimiento de las plantas habría sido casi nulo durante el período del experimento (estación invernal) dado que el crecimiento de estas plantas es principalmente estivo-otoñal (Peichotto 2010). Consideramos que la biomasa aérea es una medida más adecuada del tamaño de hábitat disponible para los artrópodos invernantes que la altura o el perímetro. Debido a que se observó sobredispersión de los datos (i.e., relación varianza/media $>1$ ) se utilizaron errores quasipoisson (Crawley 2007). Los análisis estadísticos fueron realizados con la función glm del paquete Rcmdr del programa R (versión 3.1.0; The $R$ Foundation for Statistical Computing 2014). Se evaluó el efecto de la triple interacción (i.e., Biomasa x Agrupamiento x Arquitectura) sobre los modelos estadísticos de abundancia y riqueza de artrópodos (Crawley 2007). En ninguno de estos modelos la triple interacción fue significativa $(P>0.05)$, por lo que fue eliminada de los modelos finales.

\section{Resultados}

Se capturaron 1854 individuos de 37 morfoespecies que se agruparon en 21familias, 10 órdenes y dos clases (Apéndice 1). El 70\% del total de morfoespecies determinadas (26 morfoespecies) estuvieron representadas por 5

Tabla 1. Resultados de los modelos lineales generalizados realizados para evaluar los efectos de la biomasa, el agrupamiento y la arquitectura de plantas de $S$. condensatum sobre la abundancia y riqueza de artrópodos invernantes. Los valores en negrita muestran los resultados significativos.

Table 1. Results of the generalized linear model for the effects of plant biomass, clustering and architecture of $S$. condensatum, on the abundance and species richness of wintering arthropods. Bold values denote significant results.

\begin{tabular}{llcccc}
\hline $\begin{array}{l}\text { Variable } \\
\text { dependiente }\end{array}$ & Tratamiento & $\begin{array}{c}\text { Suma de } \\
\text { cuadrados }\end{array}$ & g.l. & $\mathrm{F}$ & $\mathrm{P}$ \\
\hline Abundancia & Biomasa & 194.27 & 1.33 & 19.74 & 0.0001 \\
& Agrupamiento & 37.27 & 1.33 & 3.79 & 0.0602 \\
& Arquitectura & 74.22 & 1.33 & 7.54 & $\mathbf{0 . 0 0 9 7}$ \\
& Biomasa x Agrupamiento & 11.17 & 1.33 & 1.14 & 0.2944 \\
& Biomasa x Arquitectura & 116.84 & 1.33 & 11.87 & $\mathbf{0 . 0 0 1 6}$ \\
Riqueza & Agrupamiento x Arquitectura & 32.81 & 1.33 & 3.33 & 0.0769 \\
& Biomasa & 4.73 & 1.33 & 8.07 & $\mathbf{0 . 0 0 7 7}$ \\
& Agrupamiento & 3.74 & 1.33 & 6.39 & $\mathbf{0 . 0 1 6 5}$ \\
& Arquitectura & 0.33 & 1.33 & 0.56 & 0.4586 \\
& Biomasa x Agrupamiento & 2.77 & 1.33 & 4.63 & $\mathbf{0 . 0 3 8 7}$ \\
& Biomasa x Arquitectura & 0.66 & 1.33 & 1.13 & 0.2958 \\
& Agrupamiento x Arquitectura & 1.58 & 1.33 & 2.69 & 0.1106 \\
\hline
\end{tabular}



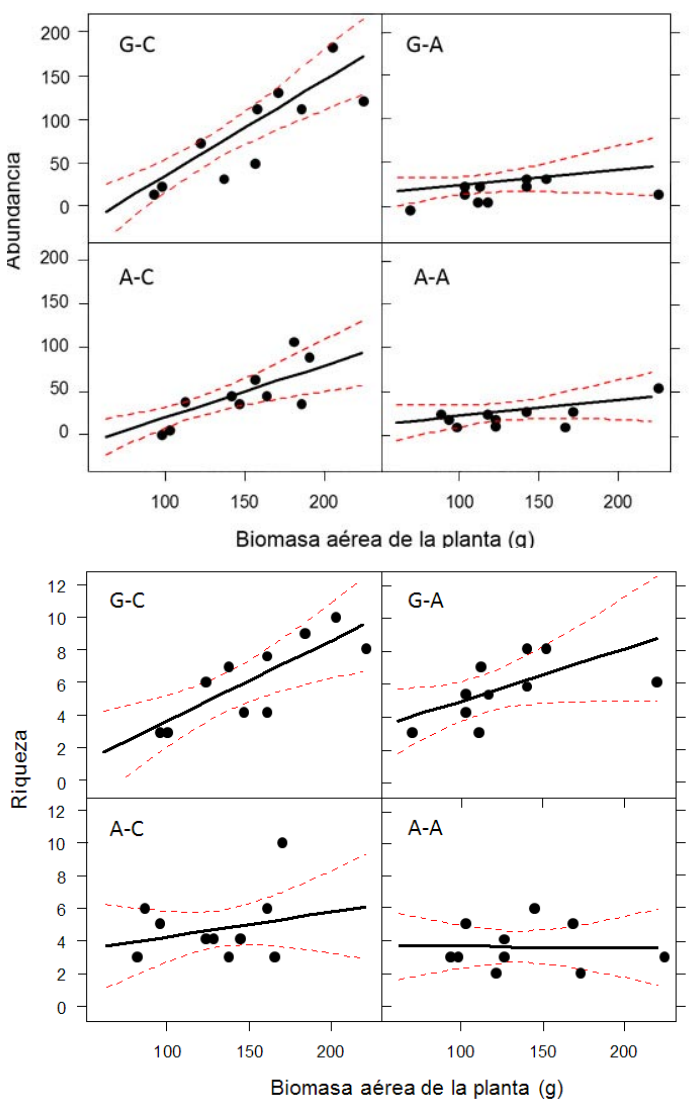

Figura 2. Abundancia (a) y riqueza (b) de artrópodos presentes en plantas de $S$. condensatum asignadas a los tratamientos de arquitectura y agrupamiento en función de la biomasa de las plantas. Línea continua: valor estimado por el modelo; línea punteada: intervalos de confianza. Puntos negros: valores observados. G-C: plantas en grupo con arquitectura cerrada, G-A: plantas en grupo con arquitectura abierta, A-C: plantas aisladas con arquitectura cerrada y A-A: plantas aisladas con arquitectura abierta.

Figure 2. Abundance (a) and richness (b) of arthropods present in S. condensatum plants allocated to architectural and clustering treatments as a function of plant biomass. Continuous line: estimated values by the model. Discontinuous line: confidence intervals. Black dots: observed values. G-C: clustered plants with closed architecture, G-A: clustered plants with open architecture, A-C: isolated plants with closed architecture, and A-A: isolated plants with open architecture.

ó menos individuos. El 91\% de la abundancia y el $33 \%$ de la riqueza total correspondieron a especies predadoras, mientras que los herbívoros alcanzaron el $8 \%$ y el $48 \%$ de la abundancia y la riqueza, respectivamente. Tanto los detritívoros como los parasitoides presentaron baja abundancia y riqueza durante el período de estudio (Apéndice 1). El orden Coleoptera fue el más abundante (90\% de la abundancia total), con dos especies predadoras de Coccinellidae afidófagos, Hippodamia convergens y Coccinella ancoralis. Cuatro especies de chinches fitófagas de la familia Pentatomidae (Edessa meditabunda,
Nezara viridula, Dichelops furcatus y Piezodorus guildinii) y un Lygaeidae del género Nysius constituyen los principales herbívoros plagas de los cultivos de la región (Apéndice 1).

$\mathrm{Al}$ inicio del experimento no se observaron diferencias significativas en altura (Agrupamiento $F_{1.32}=1.63, P=0.21$; Arquitectura $F_{132}=1.85, P=0.18$; Át grupamientoxArquitectura $F_{1,32}^{1,32}=0.59, P=0.45$ ) o perímetro (Agrupamiento $F_{1,32}^{1,32}=0.26, P=0.62$; Arquitectura $F_{1,32}=0.04$, $P=0.85$; Agrupamiento $x$ Arquitectura $\left.F_{132}=3.34, P=0.08\right)$ entre las plantas asignadas a los distintos tratamientos de arquitectura y disposición espacial. Un patrón similar se observó para la biomasa de las plantas al finalizar el experimento (Agrupamiento $F_{1,32}=0.02, P=0.90$; Arquitectura $F_{1,32}=1.47$, $P=0.23$; Agrupamiento $\mathrm{x}$ Arquitectura $\left.F_{1,32}=1.56, P=0.22\right)$.

Para la abundancia de artrópodos invernantes se observó una interacción significativa entre la biomasa y la arquitectura de las plantas (Tabla 1). En las plantas más grandes se registró más del doble de la abundancia de artrópodos en las plantas cerradas que en las abiertas, mientras que en las plantas más pequeñas no hubo efectos de la arquitectura sobre la abundancia de los mismos (Figura 2a). No hubo diferencias en la abundancia de artrópodos entre plantas agrupadas o aisladas (Tabla 1; Figura 2a).

Para la riqueza de artrópodos se detectó una interacción significativa entre la biomasa y la disposición de las plantas (Tabla 1). En las plantas pequeñas se registró similar riqueza de artrópodos entre plantas aisladas que entre plantas dispuestas en grupo, mientras que en las plantas más grandes se detectó casi el doble de especies en plantas dispuestas en grupos que en plantas aisladas (Figura 2b). No hubo diferencias en la riqueza de especies entre plantas abiertas y cerradas (Tabla 1; Figura 2b).

\section{DisCUSIÓN}

El ensamble de artrópodos invernantes presente en plantas de $S$. condensatum durante el período de estudio se caracteriza por la dominancia de dos especies de Coccinellidae predadores (Coccinella ancoralis e Hippodamia convergens) y por varias especies de herbívoros con menor abundancia. Estas especies no habían sido registradas al momento de la selección y trasplante de las plantas por lo que consideramos que estos resultados estarían 
explicando la dinámica de las poblaciones de artrópodos entre campos de cultivos y bordes. La abundancia y la riqueza de detritívoros y parasitoides es muy baja en relación a la abundancia de depredadores y herbívoros. Si bien no disponemos de estudios similares en los que se haya evaluado la abundancia de estos grupos tróficos, suponemos que los valores registrados en nuestro estudio son bajos, posiblemente debido a la sequía que se presentó en el ambiente durante el período de estudio, producto de los efectos del fenómeno climático «La Niña» (ENSO) producido entre setiembre de 2007 y julio de 2008 (Bohn et al. 2011).

Las agregaciones invernales de Coccinellidae afidófagos en gramíneas cespitosas fueron descriptas por diversos autores en el hemisferio norte (Honek 1985; Hodek \& Honek 1996; Cervantes et al. 2003; Honek et al. 2007). Las matas densas y altas de pastos de los géneros Muhlenbergia y Festuca, semejantes a las de S. condensatum en la Región Pampeana, constituyen refugios de invernación masiva de Hippodamia koebelei y Coccinella nugatoria en la Sierra Nevada mexicana (Cervantes et al. 2003). En nuestro trabajo detectamos invernación masiva de dos especies de Coccinellidae de los mismos géneros que los descriptos por Cervantes et al. (2003). Sin embargo, las matas de gramíneas no constituyen el único sitio de invernación de estas especies. En el área de estudio, C. ancoralis también fue detectada en el período invernal debajo de ritidomas de Eucalyptus camaldulensis, junto a otras siete especies de coccinélidos (Bulacio et al. 2005), y en nidos abandonados de «leñatero» (Anumbius annumbi, Furnariidae), tanto en áreas agrícolas como ganaderas (Montero \& Vignaroli 2008; Turienzo \& Di Iorio 2008). En contraste, $H$. convergens - que en valles y llanuras de California, además de hallarse en el interior de matas de gramíneas también forma agregaciones en la base de plantas perennes prominentes (Hagen 1962; Hodek \& Honek 1996) - en el área de estudio sólo fue detectada en densidades muy bajas fuera de las matas de las gramíneas.

Cuatro especies de chinches fitófagas de la familia Pentatomidae (Edessa meditabunda, Nezara viridula, Dichelops furcatus y Piezodorus guildinii, consideradas plagas de relevancia en el cultivo de soja en estado reproductivo [Massoni \& Frana 2008]) fueron encontradas en muy baja abundancia. Monetti et al. (2008) determinaron que el reingreso a la actividad de los adultos de Nezara viridula luego de su diapausa invernal se logra a fines de agosto, cuando la disponibilidad de recursos aumenta y el riesgo de heladas tardías disminuye. Mecanismos semejantes pueden haber operado bajo las condiciones benignas del invierno de 2008, adelantando la partida de estas especies desde sus sitios de invernación. La baja abundancia de chinches durante el período de barbecho puede ser consecuencia de su presencia escasa en el cultivo de soja antecesor. Por el contrario, cuando se verifican densidades altas de chinches en etapas tardías del cultivo de soja, es frecuente detectar mayor abundancia de $N$. viridula y $P$. guildinii invernando, tanto en matas de gramíneas como debajo de ritidomas de Eucalyptus, en áreas de bordes de cultivos pampeanos (Bulacio et al. 2005; Montero 2008).

\section{Efectos del tamaño, la arquitectura y el agrupamiento de la planta hospedera}

En proporción, las plantas de mayor tamaño tuvieron una mayor abundancia y riqueza de artrópodos que las más pequeñas. Observaciones similares han sido registradas en otros hábitats utilizados como refugios invernales (Cervantes et al. 2003). Las dos especies de Coccinellidae fueron más abundantes sobre plantas cerradas. Este efecto fue mayor sobre plantas cerradas dispuestas en grupos, y más importante para Coccinella ancoralis que para Hippodamia convergens. Por el contrario, en plantas abiertas, $H$. convergens presentó mayor abundancia de individuos que C. ancoralis. La elevada abundancia total de artrópodos invernantes en las plantas cerradas y la mayor riqueza total sobre las plantas agrupadas demuestran que la apertura y el aislamiento de las plantas influyen de manera negativa sobre la selección de los sitios de refugio por parte de los artrópodos invernantes. En las plantas cerradas de $S$. condensatum que crecen cercanas entre sí se dan las condiciones adecuadas para que opere la "agregación hipsotáctica», es decir, la formación de grupos densos de plantas y de una compleja red de tallos y hojas que intervienen en la estructuración de los grandes agregados de coccinélidos, donde están en contacto íntimo unos con otros, ocultándose entre sus vecinos (Hagen 1962; Honek et al. 2007). En consecuencia, al abrir la mata y modificar su arquitectura se desarma esta red y se pierde la estructura física del microhábitat, por lo que la planta no resulta adecuada para alojar estos insectos ya que no logran tocarse entre sí. Este tipo de gregarismo invernal se caracteriza por la concentración de 
individuos atraídos por estímulos quimio- o tigmotácticos encargados de activar la producción de feromonas de agregación; como consecuencia se observa un comportamiento de atracción de individuos conespecíficos hacia objetos prominentes, aislados y situados en condiciones relativamente secas (Hagen 1962; Turnock 1996; Cervantes et al. 2003; Nalepa et al. 2003; Labrie et al. 2008; Susset et al. 2013; Wheeler \& Cardé 2013). En estas agregaciones, el fitness de los individuos se relaciona de forma positiva con la densidad de conespecíficos (Stephens et al. 1999). En las plantas cerradas, las de mayor tamaño presentan proporcionalmente mayor abundancia de individuos que las más pequeñas. El efecto positivo del tamaño de la planta se diluye al abrir la misma, modificando su estructura, dado que no se darían las condiciones para la agregación hipsotáctica de los coccinélidos. Por otro lado, las plantas agrupadas resultan contrastantes y fácilmente distinguibles por las distintas especies de coccinélidos en el paisaje llano de la región Pampeana. Estos parches de mayor tamaño proporcionan sitios secos y resguardados que aumentan las probabilidades de supervivencia en los períodos climáticos adversos (Cervantes et al. 2003).

Si bien no se midieron variables microclimáticas, consideramos que las menores temperaturas, el clima lumínico intra-planta o la amplitud hídrica diaria podrían ser los factores causantes de la baja riqueza de artrópodos que invernan en las plantas abiertas.

\section{Consideraciones sobre el control natural y la} conservación en agroecosistemas pampeanos

A nivel mundial, los cambios en la forma de uso de la tierra han generado ambientes agrícolas muy simplificados en términos de biodiversidad ambiental, vegetal y animal (Ryszkowski \& Jankowiak 2002). La disminución de la biodiversidad y la consecuente homogeneización del hábitat como resultado de las prácticas culturales más utilizadas por la agricultura moderna comprometen tanto la sustentabilidad como la estabilidad y la productividad futura de los ecosistemas agrícolas (Cohen 1999; Benton et al. 2003). En los agroecosistemas pampeanos se ha producido una disminución marcada de la diversidad de cultivos agrícolas, asociada al incremento de la dominancia del cultivo de soja. Sumado a esto, la expansión de las fronteras agrícolas y la pérdida de biodiversidad por destrucción de ecosistemas naturales determina una tendencia clara hacia la homogeneización del paisaje agrícola pampeano (Altieri \& Pengue 2006; Aizen et al. 2009).

La promoción de niveles elevados de biodiversidad en los agroecosistemas puede ser un medio efectivo para mantener su funcionamiento y resiliencia frente a las perturbaciones externas (Holling 1973; Swift et al. 2004; Montero 2008). En consecuencia, la incorporación de parches de vegetación natural en las áreas de cultivo no sólo incrementa la abundancia de especies predadoras invernales, sino que estos parches se transforman en centros de dispersión de enemigos naturales durante el período primaveral (Carmona \& Landis 1999; Landis et al. 2000). Asimismo, los parches de vegetación espontánea adyacentes a los cultivos cumplen un rol importante como reservorios o refugios de algunos predadores y garantizan su presencia en las áreas de cultivo (Thomas \& Marshall 1999; Cicchino et al. 2003; Montero 2014).

La mayoría de los coccinélidos pampeanos son especies preferentemente afidófagas (Saini 1985, 2004; Montero \& Vignaroli 2008; Montero 2014); constituyen un factor de control natural de las poblaciones de pulgones que afectan a una variedad de cultivos y han sido empleados en estrategias clásicas de control biológico (Salto et al. 1990; Gyenge et al. 1998). También pueden alimentarse de desoves y de los primeros estadios larvales de lepidópteros (Molinari 1987), de trips (Dughetti 1997) o de moscas blancas (Hagler et al. 2004).

Nuestro estudio da indicios acerca de la conveniencia de mantener y promover la presencia de matas de $S$. condensatum en los bordes agrícolas pampeanos, con el objeto de proporcionar sitios seguros de refugio invernal y así facilitar la permanencia de coccinélidos afidófagos generalistas en la proximidad de los lotes de cultivo. Si bien este estudio se realizó en una sola localidad y en un año en particular, recomendaciones similares han sido realizadas en otros estudios. En el área ganadera de la Sierra Nevada mexicana se sugiere evitar el sobrepastoreo y la quema de matas densas y altas de gramíneas cespitosas ya que proporcionan condiciones adecuadas de supervivencia y permanencia para especies de coccinélidos depredadores de plagas agrícolas (Cervantes et al. 2003). La manipulación de la diversidad espacial, como es el caso de la creación de refugios 
en los bordes, bajo la forma de arboledas, tocones de cortinas forestales, setos con arbustivas, cúmulos de paja de gramíneas o matas densas de gramíneas cespitosas como $S$. condensatum, puede constituir una importante estrategia en programas de control natural y manejo integrado de plagas (Altieri 1992; Carmona \& Landis 1999; Landis et al. 2000; Lee et al. 2001).

Agradecimientos: Agradecemos a la Facultad de Ciencias Agrarias de la U.N.R. por facilitarnos las instalaciones para realizar el estudio. A dos revisores anónimos y a la Editora Adriana Ruggiero por sus sugerencias y comentarios que permitieron mejorar la calidad del manuscrito. A Darién E. Prado por revisar el inglés del abstract y de las leyendas de figuras y tablas.

\section{BibLIOGRAFÍA}

Aizen, MA; LA Garibaldi \& M Dondo. 2009. Expansión de la soja y diversidad de la agricultura argentina. Ecol. Austral, 19:45-54.

Altieri, MA \& CI Nicholls. 1999. Biodiversity, ecosystem function, and insect pest management. Pp. 197-236 en: Collins, WW \& CO Qualset (eds.). Biodiversity in Agroecosystems. CRC Press, Florida, EEUU.

Altieri, MA \& WA Pengue. 2006. La soja transgénica en América Latina. Biodiversidad, 47:14-19.

Altieri, MA. 1992. Biodiversidad, agroecología y manejo de plagas. CETAL. Santiago, Chile. Pp. 162.

Benton, GB; JA Vickery \& JD WiLsON. 2003. Farmland biodiversity: is habitat heterogeinity the key? Trends Ecol. Evol., 18:182-188.

Bocanelli, SI; EF PIRE; PS TorRes \& JP LeWIS. 1999. Cambios en la vegetación de un campo abandonado después de un cultivo de trigo. Pesq. Agropecu. Bras., 34:151-157.

Bohn, VY; MC Piccolo \& GME Perillo. 2011. Análisis de los períodos secos y húmedos en el sudoeste de la provincia de Buenos Aires (Argentina). Rev. Climatol., 11:31-43.

Borror, DJ; CA Triplehorn \& NF Johnson. 1992. An introduction to the study of insects. $6^{a}$ Ed. Sunders College Publishing, Harcourt Brace Publishers, Orlando, EEUU. Pp. 875.

BuCHER, EH. 1974. Observaciones ecológicas sobre los artrópodos del bosque chaqueño de Tucumán. Rev. Fac. Cs. Ex. Fís. Nat. Serie: Cs. Biol., 1:35-122.

Bulacio, V; GA Montero; L Vignaroli \& D Prado. 2005. Diversidad de artrópodos otoño-invernales presentes bajo el ritidoma de Eucalyptus. Rev. Soc. Entomol. Argent., 64:181-182.

CARMONA, DM \& DA LANDIS. 1999. Influence of refuge habitats and cover crops on seasonal activity-density of ground beetles (Coleoptera: Carabidae) in field crops. Environ. Entomol., 28:1145-1153.

Cervantes, JF; NE Galindo; J Romero \& A Pescador. 2003. Control biológico por conservación. Coccinélidos entomófagos en Zoquiapan, Estado de México. Sociedades Rurales, Producción y Medio Ambiente, 4:57-64.

Cicchino, AC; ME Marasas \& MF Paleologos. 2003. Características e importancia de la carabidofauna edáfica de un cultivo experimental de trigo y sus bordes con vegetación espontánea en el partido de La Plata, Pcia. de Buenos Aires. Revista de Ciencia y Tecnología, FA UNSdE, 8:41-55.
Cohen, JI. 1999. Agroecosystem quality: policy and management challenges for new technologies and diversity. Pp. 299-316 en: Collins, WW \& CO Qualset (eds.). Biodiversity in Agroecosystems. CRC Press, Florida, EEUU.

Crawley, MJ. 2007. The $R$ book. John Wiley \& Sons, Chichester, Inglaterra.

DANKS, HV. 2005. Key themes in the study of seasonal adaptations in insects: patterns of cold hardiness. Appl. Entomol. Zool., 40:199-211.

Dughetti, AC. 1997. Manejo integrado de trips en el cultivo de ajo. Pp. 223-230 en: Burda, JL. 50 Temas sobre producción de ajo. Volumen 3. EEA INTA La Consulta.

Farías, AA; L Marone \& F JaKsic. 2007. Gremios. Pp. 145170 en: Jaksic, F \& Marone, L. Ecología de comunidades. Ediciones Universidad Católica de Chile, Santiago, Chile. Pp. 336.

FlanNagan, RD; SP TAMariello; KH Joplin; RA CiKRAIRELAND; GD YocUM; ET AL. 1998. Diapause-specific gene expression in pupae of the flesh fly Sarcophaga crassipalpis. Dev. Biol., 95:5616-5620.

Gyenge, J; J Edelstein \& C SAlto.1998. Efectos de la temperatura y la dieta en la biología de Eriopis connexa (Germar) (Coleoptera: Coccinelidae). An. Soc. Entomol. Brasil, 27:345-356.

HAGEN, KS. 1962. Biology and ecology of predaceous Coccinellidae. Annu. Rev. Entomol., 7:289-326.

Hagler, JR; CG JACKSON; R IsAaCs \& SA MaChtLEy. 2004. Foraging behavior and prey interactions by a guild of predators on various lifestages of Bemisia tabaci. J. Insect Sci., 4:1-13.

Hawkins, CP \& JA Mac Mahon. 1989. Guilds: the multiple meanings of a concept. Annu. Rev. Entomol., 34:423-451.

Hodek, I \& A Honek. 1996. Ecology of Coccinellidae. Kluwer Academic Publishers, Dodrecht, Holanda. Pp. 464.

Holling, CS. 1973. Resilience and stability of ecological systems. Ann. Rev. Ecol. Syst., 4:1-23.

HoneK, A. 1985. Habitat preferences of aphidophagous Coccinellids (Coleoptera). Entomophaga, 30:253-264.

Honek, A; Z Martinková \& S PekÁr. 2007. Aggregation characteristics of three species of Coccinellidae (Coleoptera) at hibernation sites. Eur. J. Entomol., 104: 51-56.

LAbrie, G; D CoDerre \& E LuCAs. 2008. Overwintering strategy of Multicolored Asian Lady beetle (Coleoptera: Coccinellidae): cold-free space as a factor of invasive success. Ann. Entomol. Soc. Am., 101:860-866.

LANDis, DA; SD WratTen \& GM Gurr. 2000. Habitat management to conserve natural enemies of arthropod pests in agriculture. Annu. Rev. Entomol., 45:175-201.

Langellotto, GA \& RF Denno. 2004. Response of invertebrate natural enemies to complex-structured habitats: a meta-analytical synthesis. Oecologia, 139:1-10.

LAWTON, JH. 1983. Plant architecture and the diversity of phytophagous insects. Annu. Rev. Entomol., 28:23-39.

LEE, JC; FD MENALLED \& DA LANDIs. 2001. Refuge habitats modify impact of insecticide disturbance on carabid beetle communities. J. Appl. Ecol., 38:472-483.

MASSONI, FA \& JE FRANA. 2008. Fluctuación poblacional del complejo de chinches en alfalfa. Pp. 89-96 en: Trumper, EV \& JD Edelstein (eds.). Chinches fitófagas en soja. Revisión y avances en el estudio de su ecología y manejo. Ediciones INTA, Manfredi, Argentina. Pp. 190. 
MolinarI, AM. 1987. Conceptos y descripción de especies entomófagas asociadas a insectos plaga del cultivo de soja. Publicación Miscelánea EEA INTA Oliveros, 19:1-13.

Monetti, M; EV Trumper \& DH Pons. 2008. Diapausa invernal de Nezara viridula en la Provincia de Córdoba. Pp. 71-88 en: Trumper, EV \& JD Edelstein (eds.). Chinches fitófagas en soja. Revisión y avances en el estudio de su ecología y manejo. Ediciones INTA, Manfredi. Pp. 190.

Montero, GA \& LA Vignaroli. 2008. Un Coccinélido exótico (Harmonia axyridis) invade los agroecosistemas del sudeste de Santa Fe. Agromensajes, 26:3-4. http: //www.fcagr.unr.edu.ar/Extension/Agromensajes / 26/1AM26.htm

Montero, GA. 2008. Comunidades de artrópodos en vegetación de áreas no cultivadas del sudeste de Santa Fe. Tesis de Maestría en Manejo y Conservación de Recursos Naturales. Facultad de Ciencias Agrarias. Universidad Nacional de Rosario. Pp. 206.

Montero, GA. 2014. Ecología de las interacciones entre malezas y artrópodos. Capítulo X. Pp. 267-305 en: Fernández, OA; HA Acciaresi \& ES Leguizamón (eds.). Malezas e Invasoras de la Argentina: ecología y manejo. Tomo I. Editorial Universidad Nacional del Sur, Bahía Blanca. Pp. 964.

Morrone, JJ \& S Coscarón. 1998. Biodiversidad de Artrópodos Argentinos. Ediciones Sur. La Plata, Argentina. Pp. 599.

Musolin, DL \& H Numata. 2004. Late-season induction of diapause in Nezara viridula and its effect on adult coloration and post-diapause reproductive performance. Entomol. Exp. Appl., 111:1-6.

Nalepa, CA; GC Kennedy \& C Brownie. 2003. Orientation of Multicolored Asian Lady beetles to buildings. Am. Entomol., 50:165-166.

Pегсното, MC. 2010. Revisión taxonómica de las especies del género Schizachyrium (Poaceae: Andropogoneae) de Sudamérica. Candollea, 65:301-346.

Регсното, MC. 2003. Anatomía foliar de las especies de Schizachyrium (Poaceae: Andropogoneae) que viven en Argentina y países limítrofes. Bol. Soc. Arg. Bot., 38: 277-293.

Rizzo, HF. 1976. Hemípteros de interés agrícola. Chinches perjudiciales y chinches benéficas para los cultivos. Hemisferio Sur. Buenos Aires. Pp. 69.

Romero, GQ \& J Vasconcellos-Neto. 2005. The effects of plant structure on the spatial and microspatial distribution of a bromeliad-living jumping spider (Salticidae). J. Animal Ecol., 74:12-21.

Root, RB. 1973. Organization of plant-arthropod association in simple and diverse habitats: the fauna of collards (Brassica oleracea). Ecol. Monogr., 43:95-123.

RYSZKOWSKI, L \& J JANKOWIAK. 2002. Development of agriculture and its impact on landscape functions. Pp. 9-27 en: Ryszkowski, L (ed.). Landscape ecology in agroecosystems management. CRC Press, Washington, EEUU.

Sacchi, O; N Dalla Marta; M Constanzo \& A Coronel. 2002. Caracterización de las precipitaciones en la localidad de Zavalla. Rev. Inv. FCA-UNR, 2:91-103.
SAINI, ED. 1985. Sobre dos coccinélidos (Coleoptera) predadores de pulgones. Rev. Soc. Entomol. Arg., 44: 37- 39.

SAINI, ED. 2004. Presencia de Harmonia axyridis (Pallas) (Coleoptera: Coccinellidae) en la provincia de Buenos Aires. Aspectos biológicos y morfológicos. RIA, 33: 151-160.

Salto, C; I Bertolaccini; J Imwinkelried \& J Frana.1990. Parámetros biológicos e influencia de los distintos niveles alimenticios en el desarrollo de Eriopis connexa Guerin (Coleoptera: Coccinelidae). Publicación Técnica EEA Rafaela INTA No 50. Pp. 13.

StEPHENS, PA; WJ SUtHERLAND \& RP FRECKLETON. 1999. What is the Allee effect? Oikos, 87:185-190.

StuntZ, S; C Ziegler; U Simon \& G ZotZ. 2002. Structure and diversity of the arthropod fauna within three canopy epiphyte species in Central Panama. J. Trop. Ecol., 18: 161-176.

Susset, EC; F Ramón-Portugal; JL Hemptinne; SY Dewhirst; MA BIRKETT \& A MAGRO. 2013. The role of semiochemicals in short-range location of aggregation sites in Adalia bipunctata (Coleoptera, Coccinellidae). Chem. Ecol., 39: 591-601.

SWIFT, MJ; AM IZAC \& M VAN NOORDWIIK. 2004. Biodiversity and ecosystem services in agricultural landscape: are we asking the right questions? Agric. Ecosyst. Environ., 104:13-134.

The R foundation for Statistical Computing. 2014. R version 3.1.0

ThOMAS, CFG \& EJPMARshall. 1999. Arthropod abundance and diversity in differently vegetated margins of arable fields. Agric. Ecosyst. Environ., 72:131-144.

TuRIENZO, P \& O Di Iorio. 2007. Insects found in birds' nests from Argentina. Part I: a bibliographical review, with taxonomical corrections, comments and a hypothetical mechanism of transmission of cimicid bugs. Zootaxa, 1561:1-52.

TuRIENZO, P \& O Di IORIO. 2008. Insects found in birds' nests from Argentina: Anumbius annumbi (Vieillot, 1817) (Aves: Furnariidae). Zootaxa, 1871:1-55.

Turnock, B. 1996. Lady beetles on the Lake Manitoba beach. UFS (Delta Marsh) Annu. Report, 31:40-42.

WheELER, CA \& RT CARDÉ. 2013. Defensive allomones function as aggregation pheromones in diapausing ladybird beetles, Hippodamia convergens. Chem. Ecol., 39:723-732.

YANES, J; BO CARTWRIGHT; RD EIKERNBARY \& WD WARDE. 1982. Preference for medium dense grass tussocks by Hippodamia convergens (Col.: Coccinellidae). Entomophaga, 27: 55-59.

ZaCCAGNINI, ME; J DeCARRE; A GoIJMAn; L SOLARI; R SuÁREZ; ET AL. 2007. Efecto de la heterogeneidad ambiental de terrazas y bordes vegetados sobre la biodiversidad animal en campos de soja en Entre Ríos. Pp. 159-171 en: Caviglia, OP; OF Paparotti \& MC Sasal (eds.). Agricultura Sustentable en Entre Ríos, Ediciones INTA, Buenos Aires. Pp. 232. 


\section{INFORMACIÓN SUPLEMENTARIA}

Apéndice 1. Abundancia y riqueza de morfoespecies de artrópodos invernantes ordenados por grupos tróficos en plantas de $S$. condensatum en función del agrupamiento y la arquitectura de las plantas en la localidad de Zavalla (SF). G-C: plantas en grupo con arquitectura cerrada, G-A: plantas en grupo con arquitectura abierta, A-C: plantas aisladas con arquitectura cerrada y A-A: plantas aisladas con arquitectura abierta.

Appendix 1. Abundance and richness of arthropod morphospecies arranged by trophic groups recorded inside S. condensatum plants in Zavalla (Santa Fe), as a function of plant clustering and architecture. G-C: clustered plants with closed architecture, G-A: clustered plants with open architecture, A-C: isolated plants with closed architecture, and A-A: isolated plants with open architecture.

\begin{tabular}{|c|c|c|c|c|c|c|c|c|c|c|}
\hline $\begin{array}{l}\text { Grupo } \\
\text { Funcional }\end{array}$ & Clase & Orden & Familia & $\begin{array}{l}\text { Especie/ } \\
\text { Morfoespecie }\end{array}$ & Estado & Total & $\mathrm{G}-\mathrm{C}$ & G-A & $\mathrm{A}-\mathrm{C}$ & A-A \\
\hline \multirow[t]{3}{*}{ Detritívoros } & Insecta & Diptera & Ceratopogonidae & Ceratopogonidae sp. & Adulto & 3 & & 2 & 1 & \\
\hline & & Dictyoptera & Pseudophyllodromidae & Chorisoneura sp. & Adulto & 5 & & 1 & 3 & 1 \\
\hline & & Psocoptera & Ectopsocidae & Ectopsocidae sp. & Adulto & 4 & 4 & & & \\
\hline Herbívoros & & Hemiptera & Lygaeidae & Nysius simulans & Adulto & 3 & & 1 & 2 & \\
\hline \multirow[t]{4}{*}{ Plagas } & & & Pentatomidae & Dichelops furcatus & Adulto & 8 & 4 & & 2 & 2 \\
\hline & & & & Edessa meditabunda & Adulto & 9 & 4 & 3 & 1 & 1 \\
\hline & & & & Nezara viridula & Adulto & 10 & 6 & 1 & 2 & 1 \\
\hline & & & & Piezodorus guildinii & Adulto & 3 & 1 & & 2 & \\
\hline Herbívoros & & Hemiptera & Lygaeidae & Lygaeidae sp. 1 & Adulto & 28 & 14 & 12 & 1 & 1 \\
\hline \multirow[t]{11}{*}{ Neutrales } & & & & Lygaeidae sp. 2 & Adulto & 1 & & 1 & & \\
\hline & & & Pentatomidae & Pentatomidae sp. 1 & Adulto & 1 & & & 1 & \\
\hline & & Coleoptera & Curculionidae & Listroderes apicalis & Adulto & 1 & & 1 & & \\
\hline & & & & Curculionidae sp. & Adulto & 10 & 3 & 4 & 2 & 1 \\
\hline & & & Chrysomelidae & Chrysomelidae sp. & & 1 & 1 & & & \\
\hline & & Hymenopter & aHalictidae & Agapostemon sp. & Adulto & 1 & 1 & & & \\
\hline & & Hemiptera & Cicadidae & Cicadidae sp. & Adulto & 1 & & & & 1 \\
\hline & & & Coreidae & Coreidae sp. & Adulto & 2 & 2 & & & \\
\hline & & Lepidoptera & Arctiidae & Eurota herrichi & Adulto & 47 & 23 & 12 & 8 & 4 \\
\hline & & & ??? & Lepidoptera sp. 1 & Adulto & 3 & 2 & & 1 & \\
\hline & & & ??? & Lepidoptera sp. 2 & Larva & 2 & & & & 2 \\
\hline \multirow[t]{4}{*}{ Parasitoides } & & Hymenopter & Chalcicidae & Chalcicidae sp. & Adulto & 1 & 1 & & & \\
\hline & & & Eurytomidae & Eurytomidae sp. & Adulto & 3 & & & 1 & 2 \\
\hline & & & Ichneumonidae & Ichneumonidae sp. 1 & Adulto & 2 & 1 & 1 & & \\
\hline & & & & Ichneumonidae sp. 2 & 2. Adulto & 1 & & & 1 & \\
\hline \multirow[t]{15}{*}{ Predadores } & & Hemiptera & Reduviidae & Reduviidae sp. & Adulto & 3 & 2 & & 1 & \\
\hline & & Hymenopter & ??? & Hymenoptera sp. & Adulto & 1 & 1 & & & \\
\hline & & Coleoptera & Coccinellidae & Coccinella ancoralis & Adulto & 770 & 487 & 58 & 165 & 60 \\
\hline & & & & Cycloneda sanguínea & Adulto & 3 & 2 & & 1 & \\
\hline & & & & Eriopis connexa & Adulto & 1 & & 1 & & \\
\hline & & & & $\begin{array}{l}\text { Hippodamia } \\
\text { convergens }\end{array}$ & Adulto & 894 & 344 & 153 & 214 & 183 \\
\hline & & & & Scymnus argentinicus & Adulto & 1 & & & 1 & \\
\hline & & Dermaptera & Forficulidae & Doru lineare & Adulto & 1 & 1 & & & \\
\hline & \multirow[t]{7}{*}{ Arachnid } & la Araneae & Salticidae & Salticidae sp. 1 & Adulto & 3 & & 1 & 1 & 1 \\
\hline & & & & Salticidae sp. 2 & Adulto & 1 & & & & 1 \\
\hline & & & & Salticidae sp. 3 & Adulto & 5 & 1 & 2 & 2 & \\
\hline & & & Zodariidae & Zodariidae sp. 1 & Adulto & 9 & 5 & 3 & 1 & \\
\hline & & & & Zodariidae sp. 2 & Adulto & 6 & & 5 & & 1 \\
\hline & & & & Abundancia total & & 1854 & 910 & 264 & 416 & 264 \\
\hline & & & & Riqueza total & & 37 & 22 & 19 & 23 & 16 \\
\hline
\end{tabular}

